\title{
The Role of Dance in the Functioning and Socialization of People with Cerebral Palsy: a Pilot Clinical Trial
}

\author{
Beatriz Menezes DeJesus ${ }^{1}$, Lavinia Teixeira-Machado ${ }^{2}$
}

${ }^{1}$ Physical Therapist, Department of Physical Therapy, Federal University of Sergipe, Lagarto (SE), Brazil

${ }^{2}$ Ph.D., Physical Therapist Department of Education in Health, Federal University of Sergipe, Lagarto (SE), Brazil

\section{Type of article: Original}

\begin{abstract}
Background: Dance fosters a distinctive body language that enables communication by awakening sensations, emotions and thoughts that are fundamental to the listening body in various prisms. Dance practice can promote neuropsychomotor improvement, as well as fundamental attributes for social interaction and functional independence, in people with Cerebral Palsy (CP).

Objective: The purpose of this study was to determine the effectiveness of a specific dance program to promote body functioning and socialization of people with $\mathrm{CP}$ and how a systematization of dance protocol directed toward body dysfunctions is able to unlock the social potential inherent in every individual.

Methods: This pilot clinical trial was conducted on seven patients who have cerebral palsy were recruited at the Federal University of Sergipe, Lagarto city, Brazil, from January 03, 2019 to December 12, 2019. A specific dance program lasting 60 minutes was used. Evaluations were carried out before and after 12 months of intervention using instruments that assess dance efficiency, functional independence and social function in all participants. For statistical analysis, IBM-SPSS version 22 was used to apply the Shapiro-Wilk, Levene and Wilcoxon Matched Pars test.

Results: After 12 months of intervention, those with CP who participated in the specific dance project and public presentations were evaluated. In the Functional Independence Measure, the locomotion value (T1: 6.71 $\pm 1.94 ; \mathrm{T} 2$ : $8.20 \pm 1.93$ (95\% CI, $\mathrm{p}=0.03)$, communication (T1: $9.14 \pm 2.71$; T2: $13.00 \pm 1.00(95 \% \mathrm{CI}, \mathrm{p}=0.04)$, social cognition (T1: $9.00 \pm 2.19 ; \mathrm{T} 2: 18.00 \pm 1.51(95 \% \mathrm{CI}, \mathrm{p}=0.01)$ and total value (T1: $74.40 \pm 14.15 ; \mathrm{T} 2: 86.20 \pm 14.54(95 \% \mathrm{CI}$, $\mathrm{p}=0.008$ ) were significant, and the difficulties in highlighting social participation decreased from $57 \%$ to $26 \%$ $(95 \% \mathrm{CI}, \mathrm{p}=0.01)$.

Conclusion: Dance stimulates and contributes to social participation and functional independence through bodily dialogue. Individuals with $\mathrm{CP}$ can be encouraged neuropsychomotor, socio-educational and psychosomatic practice.

Clinical Trial Registration: The study is registered with the "Thai Clinical Trial Registry (Identification number: TCTR20200502002).

Funding: The study was developed at the Federal University of Sergipe, Brazil. This study was funded by the Coordenação de Aperfeiçoamento de Pessoal de Nível Superior - Brazil (CAPES). Finance code number 400075/2017-2, process number: 23038.011152/2017-62.

Keywords: Dance, Socialization, Cerebral palsy, Body Functioning, Communication
\end{abstract}

Abbreviations / Acronyms:

CP: Cerebral Palsy; FIM: Functional Independence Measure; KAP: Knowledge, Attitude and Practice; TALT: Técnica Aplicada Lavinia Teixeira; WHODAS: World Health Organization Disability Assessment Schedule

\section{Corresponding author:}

Beatriz Menezes DeJesus, Department of Physical Therapy, Federal University of Sergipe, Lagarto (SE), Brazil. Tel: +55.79-999862560, E-mail: beatriz.mj.bm@academico.ufs.br and fisio.beatriz19@gmail.com

Received: April 15, 2020, Accepted: November 04, 2020, Published: December 2020

Funding / research project approval: Finance code number 400075/2017-2, process number: 23038.011152/2017-62.

Ethics approval: Federal University of Sergipe (Ref: 06154012.4.0000.0058, approval number 1.056.806)

(C) 2020 The Authors. This is an open access article under the terms of the Creative Commons Attribution-NonCommercialNoDerivs License, which permits use and distribution in any medium, provided the original work is properly cited, the use is noncommercial and no modifications or adaptations are made. 


\section{Introduction}

Cerebral Palsy (CP) is well known in the positivist human biomedical area for being a neurological condition defined by a group of disorders that are located in the development of body posture and movement arising from damage to the Central Nervous System (CNS) in the early years of life. This condition impacts the child's development in the production of motor tasks, including speech articulation, and this is accompanied by learning disabilities arising from sensory disorders and cognitive behavioral changes that influence the physical, emotional and social aspects of the individual throughout their lives (1-3).

In terms of the physical aspects, changes here are due to neurological damage that impedes the mobility and independence of the individual. This has a direct influence on their quality of life as both mobility and independence affect the individual's cognitive, emotional, physical and social state (2, 4). According to Lipscombe et al. (5), deficits related to social participation affect the individual's ability to socialize and their perception of socialization; this then makes the barriers of social rejection even harder to overcome. Added to this is the fact that functional independence, which is impaired by $\mathrm{CP}$, is an essential factor for social interaction, as movement is vital for non-verbal communication, and also enables freedom of movement, which is considered to be a fundamentally human entitlement. Motor disability consequently limits the possibilities for communication $(3,5,6)$. It is therefore necessary to develop interventions aimed at improving cognitive-behavioral functionality, including the promotion of social interaction to boost self-confidence and self-esteem.

One possible intervention in CP to promote both functionality and social interaction is dance practice, as it has the ability to expand the body's potential through sensory, emotional and neuromotor processes. Dancing promotes functional independence, as it stimulates static and dynamic body balance; this is one of the principles of psychomotor learning because it reveals the body's ability to think and represent a movement, as well as promoting body perception (7). In addition, dance awakens body discourse through non-verbal communication, and thus aids neuropsychomotor development, physical and emotional well-being, education and body perception, self-confidence and self-esteem, social interaction and functional independence $(8,9)$. In terms of the social aspects, Agamben (10) explains that negative thoughts impair an individual's potential ability to perform certain tasks. During a dance intervention, it is possible for different bodies, rhythms and movements to express the art of dance without necessarily having the perfect body and motor skills that are dictated by social norms (7). Based on the above, the aim of this study was to determine the effectiveness of a specific dance program in benefiting socialization and functional independence in people with CP, and, as a secondary outcome, to demonstrate how the systematization of dance practice in psychomotor dysfunctions can influence neuropsychomotor improvement in CP.

\section{Material and Methods}

\subsection{Study design and participants}

This study was a pilot clinical trial. It addresses dance practice during 12 months as an option for body discourse, which contributes to body perception and education, social interaction and functional independence in children and adolescents with CP. Individuals with CP who participated in the TALT group (Técnica Aplicada Lavinia Teixeira) and who were aged between 5 and 25 years were included. Those who did not participate in classes and / or assessments were excluded.

\subsection{Assessment}

First, parents and/or caregivers signed the informed consent form which presented the objectives, proposed procedures and expected benefits. Subsequently, the first assessment of all the participants was performed, with responses by the caregiver if the child or adolescent was not able to respond for themselves for any reason (for example, cognitive impairment or aphasia) (1,3). After signing the consent form, another form was completed to register the participants' important personal data (name, age, gender, weight, height, body mass index, professional occupation, marital status, name of the person responsible, telephone number for contact) and anamnesis (current, past, physiological, social, family and medication history).

To assess the impact of dance on functionality and socialization, we used the following assessment instruments at two different time-points: at the beginning of the study (T1) and after 12 months (T2) of dance practice. To assess the effectiveness of dance practice we applied the Knowledge, Attitude and Practice Survey (KAP). This is a semistructured interview based on questions about knowledge, thinking and acting on actions by parents or caregivers before and during this study protocol. The KAP interview can offer a diagnosis of achievements in the educational, emotional and social dimensions that demonstrate the effectiveness of an intervention (11). To assess body functioning 
we used the Functional Independence Measure (FIM), a scale that assesses functionality at different levels with or without help, verifying the individual's performance in 18 activities in the physical, cognitive and social domains. Rating is numerical: "1" represents total help; "2" maximum help; "3" moderate help; "4" minimum help; "5" supervision; "6" independence with technical help; and "7" complete independence. The FIM is divided into central themes, namely: [A-F] Self-care; [G-H] Sphincter Control and Hygiene; [I-K] Mobility; [L-M] Locomotion; [N-O] Communication; and finally, [P-R] Social Cognition. The range varies between 18 and 126 points, and the higher the score, the higher the level of functional independence $(12,13)$.

To analyze social function, we used the World Health Organization Disability Assessment Schedule (WHODAS), which corresponds to a practical instrument for social participation because it does not have the specific clinical condition as a focus, but rather a form of health and disability assessment, providing a level of functionality. It is directly related to the International Classification of Functioning, Disability and Health (ICF), not clearly distinguishing measures of symptoms and disability. The score obtained from the WHODAS (2.0 version) scale ranges from 0 to 4: the individual with a score of " 0 " has no difficulty for a given action; "1" represents mild difficulty; " 2 " moderate difficulty; " 3 " severe difficulty and "4" extreme difficulty or inability to perform $(14,15)$.

\subsection{Dance Protocol}

The dance classes were based on the concepts and proposals developed in the TALT (Técnica Aplicada Lavinia Teixeira) program, and took place twice per week, each session lasting 60 minutes, over a period of twelve months, in an appropriate room, alongside other dancers with or without disabilities. The educational focus was to carry out choreographic sequences in a playful way to promote socialization, body balance, functional independence, motor coordination, and neuropsychomotor development. Each dance class consisted of three stages: (I) warm-up: stretching and body conditioning; (II) choreographed sequences: encouragement of rhythm and body perception, choreographic montages for public presentations; (III) relaxation. As follows, three axes of components underlie the TALT technique: the self, the environment, and the other:

\subsubsection{The self}

The practices that make up this axis are based on: (1) body image (mental representation that includes affective, cognitive and social aspects); (2) range of movements (represented by the planes and axes of movement for the use of the body in its full angular range); (3) motor coordination (implicit bilateral interactions in visomotor movements); and (4) ability and agility of movements (execution of bilaterally varied movements, exploring mechanisms of motor coordination and body and postural stability, with simultaneous activity of segments with and without alternation of upper and lower limbs) $(6,16,17)$.

\subsubsection{The environment}

Classroom with appropriate floor, a mirror and a stereo. It is better if the room in which the dance class takes place does not contain a lot of sensory information, such as noises (the volume of the music must not be loud), bright colors on the walls, paintings, lamps, or other devices that may distract the participants' attention. In order to trigger body image, range of motion, motor coordination and agility of movement, the dance class must contain Laban's planar levels (low, medium and high), the movements during the dance steps occur in all directions (front to back, side to side, and diagonals). In addition, devices and props should be used to enhance communication in dance, such as tambourines, rattles, fabric $(16,18-21)$.

\subsubsection{The other}

The positioning of dance class members in a circle enables interpersonal experiences and intercorporeal dialogue. The attitude embodied during dance duets is taken as an example of active non-verbal attunement between partners during performance interaction. Choreographic performances trigger the sense of intersubjectivity and differentiation of self and otherness through shared and synchronized features of motion. Public presentations emphasize the naturalness of a human being in the world with others and of being perceived as the center of expectation by others who have not experienced the dance class themselves. This contributes to the important properties of engagement and motivation, which can be considered as a mutual phenomenon between the individual and the other/others. This condition greatly influences the social being, who is mostly on the sidelines unaware that his/her actions can be reflected in the other this can come about through the eloquent clapping of the audience, as he/she perceives themselves as a central actor, with a positive character. This in turn has an effect on self-esteem. 


\subsection{Research ethics}

The Ethics Committee on Research with Human Beings of the Federal University of Sergipe under CAAE (Certificado de Apresentação de Apreciação Ética) number 06154012.4.0000.0058 (approval number 1.056.806) approved the assessment and intervention methods used in this study. Patients and parents and/or caregivers were aware of the research and its objectives, but they were not directly involved in the design of this study. Parents and/or caregivers signed the informed consent form which presented the objectives, proposed procedures and expected benefits.

\subsection{Statistical analysis}

The variables are presented with measures of mean and standard deviation - Mean (SD). All tests were performed using IBM@ SPSS $\odot$ Statistics version 22 (IBM@ Corp., Armonk, NY, USA). All analyzes were conducted using data from participants who completed the post-intervention assessment (e.g. endpoint assessment). Thus, participants without data recorded for a given parameter were automatically excluded from the analysis of that parameter. To verify the normality of the data, the Shapiro-Wilk test was applied. Homogeneity was calculated from the Levene test. Wilcoxon Matched Pars test analyzed baseline differences. Comparisons between value pairs where the global test reached a significance level of $\mathrm{p}<0.05$ (two-tailed) were corrected for multiple comparisons using Bonferroni correction.

\section{Results}

Seven children and adolescents with a clinical diagnosis of CP underwent the study, four female and three male, with an average age of 17.28 (6.87) years. After 12 months of classes and some public dance performances, re-assessments were performed. In the FIM (Functional Independence Scale), the total values increased from 74.40 (14.15) to 86.20 (14.54). This increase represents a development in functional independence. The indicators that showed the most progress was in locomotion $(\mathrm{p}=0.03)$, communication $(\mathrm{p}=0.04)$ and social cognition $(\mathrm{p}=0.01)$, as seen in Table 1 . Regarding social participation, the WHODAS values ranged from $57 \%$ to $26 \%$, meaning that there was a reduction in the difficulties faced in some situations for social interaction.

Table 1. Functional Independence Measure (FIM) values, before and after dance classes, over a period of twelve months.

\begin{tabular}{|l|l|l|l|}
\hline MIF Domains & BEFORE & AFTER & p-value \\
\hline Self-Care & $23.00(5.19)$ & $23.00(5.70)$ & 0.31 \\
\hline Sphincter Control & $12.00(2.19)$ & $12.00(2.00)$ & 0.36 \\
\hline Mobility & $10.66(3.90)$ & $12.00(3.83)$ & 0.17 \\
\hline Locomotion & $6.71(1.94)$ & $8.20(1.93)$ & $0.03^{*}$ \\
\hline Communication & $9.14(2.71)$ & $13.00(1.00)$ & $0.04^{*}$ \\
\hline Social Cognition & $9.00(2.19)$ & $18.00(1.51)$ & $0.01^{*}$ \\
\hline Total Value & $74.40(14.15)$ & $86.20(14.54)$ & $0.008^{*}$ \\
\hline
\end{tabular}

Mean (SD). Wilcoxon Matched Pars Test for Dependent Variables

The fundamental finding was the adherence of the participants to the dance program, and the parents' and/or caregivers' confidence in the proposal suggested by the study. With regard to the KAP survey, most showed no knowledge of the clinical condition of $\mathrm{CP}$, but reported it to be "a lack of oxygen in the brain" and "paralysis of some limbs". The initial attitude of all individuals was to seek treatment in a therapeutic form, for example, dance, physical therapy, speech therapy, horse therapy, and neurological monitoring. Over time, the children and adolescents showed more involvement with the members of the dance program, as well as being more willing to participate and engage in public performances, thus verifying the impact of dance on autonomy, self-confidence and self-esteem.

\section{Discussion}

In terms of functional independence, participants improved in overall scores in FIM and in the locomotion, communication and cognition domains. After 12 months, social participation increased, probably as a result of taking part in a regular activity in a social environment, as well as performing choreographies in public spaces, such as theaters, schools and convention centers. As in the studies by Teixeira-Machado \& DeSantana (7) and TeixeiraMachado, Azevedo-Santos, DeSantana (4), we reported that dance practice positively influenced functional capacity, emotional aspects and social interaction in individuals with physical limitations. With regard to physical aspects, the main focus of dance is body awareness rather than feeling as a function that underlies the perception of the body in spacetime, without excluding the influence of weight and the flow of movement. Laban $(1,8)$ highlights four primordial pillars for the movement of dance: space, time, weight and fluency that generate the internal participation 
of attention, intention, decision and progression, affecting the human faculties of thinking, perceiving, intuiting and feeling. Therefore, the focus of dance is on body movement in different forms of individual creative movements that enable the acquisition of practical motor skills due to the dialogical action of body listening and internalized movement; this is based on movement of expression arising from the actual organization that comes from thoughtbody-action. According to Koch et al. (22), dance can develop positive effects on affection, well-being, body image and mood. Taking into account the reports of people with everyday experience of the participants of this study, i.e. their parents and caregivers, who revealed the impact of dance practice on their mood, reflecting affectivity. They noticed behavioral changes in the children during dance classes that were also seen in other social spaces that the participants attended, such as school and family environments. It is noteworthy that parents themselves feel trapped by society's judgment, and even they often do not believe that their child is capable of dancing or performing on a stage, for example. The dance program envisioned in this study has gone some way in overcoming the stigma surrounding the ability of people with disabilities (PWD) to express themselves through their bodies and perform on stage. Laban (18) states that: "A simple gesture from any part of the body reveals an aspect of our inner life." Bearing this in mind, dance can foster body expression, enabling the collective participation of various corporal conditions in the same social context. Thus, the ability of the participants in this study to dance and express their emotions through facial expressions, body involvement and group energy is ratified, especially the impact of the audience's warm applause. According to Le Breton (23), social status is dependent on the representations of the body and worldview, a person definition. Thus, dance is a practice that integrates and provides the inclusion of being in the world that is given to it, encompassing personal, social, cultural, emotional, cognitive and psychological factors. Dance practice promotes the recognition of the individual's place in a society that is rightfully their own. Through self-confidence, autonomy and self-esteem they are able to tackle the barriers imposed by society on their social condition.

It is also possible to relate Foucault's (24) discourse with the lived reality, showing that the prejudiced society hinders the social interaction of people with $\mathrm{CP}$, as they have a principle of exclusion through rejection and prohibition to the different. Durkheim (25) says the body expresses one's autonomy without following the cultural and social environment in which it lives. This becomes an important statement for dance performed by the PWD, for instance, on a stage the stigmatized physical condition supersedes the disability paradigm. Analogously to the mathematics cited by Foucault (24) in the work "The Order of Discourse", metaphorically, dance can be a method of social interaction for PWD that is adverse to the arithmetic of society, that is, to eliminate inequality and insert them into social geometry as a way of overcoming barriers and providing equality within society. We can then allude to the psycho-sociological taxonomy of body techniques elucidated by Marcel Mauss (26) about what is expected of a person with disability. Although Mauss26 did not elucidate contextualization about PWD, he describes the term "technique" as an effective traditional act, the body being the first and most natural and, at the same time, technical means. More than this, everything in us is imposed by the contest between body and moral and intellectual symbols. In this way, dance practice inexorably promotes a series of acts of physical-socio-psychological montage and suppresses the sociopsychological marginalization of the stigmatized person, since social influences substantially affect the human body defined by social and cultural experiences. Moreover, artistic presentation can break institutionalized precepts in society and encompass a different reality through body movement, a fact that fosters social and cognitive performance in PWD, reflecting in their functional independence. The limitation of study lies in the low number of participants with this clinical condition. Therefore, further studies are needed to demonstrate the effects of dance on functionality in people with CP from the psychosocial aspect.

\section{Conclusions}

These feasible findings show that dance is an encouraging and facilitating option for social interaction and functional independence, as it offers a chance for body dialogue, autonomy, self-confidence, self-esteem, physical and emotional well-being, fundamental to health promotion and quality of life in people with CP. Studies with more consistent methodological designs and with a larger number of participants are needed, so that we can identify the role of dance in the functioning and socialization of people with Cerebral Palsy.

\section{Acknowledgments:}

The authors thank the Coordenação de Aperfeiçoamento de Pessoal de Nível Superior (CAPES) for funding the research, and all research participants, their families who accompanied and encouraged during this period and all volunteers during the development of the research.

\section{Conflict of Interest:}

There is no conflict of interest to be declared. 


\section{Authors' contributions:}

Both authors contributed to this project and article equally. Both authors read and approved the final manuscript. Conception or design of the work (LTM), Acquisition of data (both authors), Analysis or interpretation of data (both authors), Drafting the manuscript (both authors), Revising the manuscript (both authors), Revising the manuscript (both authors), Accountable for all aspects of the work (both authors).

\section{References:}

1) Camargos ACR, Lacerda TTB, Barros TV, Silva GC, Parreiras JT, Vidal THJ. Relationship between functional independence and quality of life in cerebral palsy. Fisioterapia \& Movimento. 2012; 25(1):83-92. Doi: 10.1590/S0103-51502012000100009

2) Ho PC, Chang CH, Granlund M, Hwang AW. The Relationships Between Capacity and Performance in Youths With Cerebral Palsy Differ for GMFCS Levels. Pediatr Phys Ther. 2017; 29(1):23-9. Doi: 10.1097/PEP.0000000000000332, PMid: 27984462

3) Matsukiyo A, Goh AC, Asagai Y. Relationship between muscle-tendon length, range of motion, and resistance to passive movement in children with normal and increased tone. The Journal of Physical Therapy Science. 2017; 29(2):349-55. Doi: 10.1589/jpts.29.349, PMid: 28265172, PMCid: PMC5333003

4) Teixeira-Machado L, Azevedo-Santos I, DeSantana JM. Dance improves functionality and psychosocial adjustment in cerebral palsy: a randomized controlled clinical trial. American Journal of Physical Medicine \& Rehabilitation. 2017; 96(6):424-9. Doi: 10.1097/PHM.0000000000000646, PMid: 27820729

5) Lipscombe B, Boyd RN, Colemen A, Fahey M, Rawicki B, Whittingham K. Does early communication mediate the relationship between motor ability and social function in children with cerebral palsy? Research in Developmental Disabilities. 2016; (53-54):279-86. Doi: 10.1016/j.ridd.2016.02.013, PMid: 26955913

6) Teixeira-Machado L, DeSantana JM. Effect of dance on lower-limb range of motion in young people with cerebral palsy: a blinded randomized clinical trial. Adolesc Health Med Ther. 2019; 10:21-8. Doi: 10.2147/AHMT.S177867, PMid: 30988649, PMCid: PMC6441460

7) Teixeira-Machado L, DeSantana JM. Dance therapy and quality of life of people with disabilities: controlled clinical trial. Revista Brasileira de Qualidade de Vida. 2013; 5(1):39-52. Doi: 10.3895/S217508582013000100005

8) Teixeira-Machado L, DeSantana JM. Dance Therapy improves quality of life in individuals with neuromotor disorders: randomized controlled trial. International Journal of Humanities Social Sciences and Education. 2015; 2(4):84-92.

9) Garcez PA, Teixeira-Machado L, DeSantana JM. Ballet Dance Portrays the Balance and Coordination Duality in Cerebellar Ataxia: A Case Report. International Journal of Scientific Engineering and Applied Science. 2016; 2(5).

10) Agamben G. Potentialities: collected essas in Phylosophy. Stanford University Press, Stanford. $2000 ; 328$.

11) Ribeiro KFC, Moura MSS, Brandão RGC, Nicolau AIO, Aquino PS, Pinheiro AKB. Knowledge, Attitude and Practice of nursing students about the Pap smear. Texto \& Contexto Enfermagem. 2013; 22(2):460-7. Doi: 10.1590/S0104-07072013000200023

12) Riberto M, Miyazaki MH, Jucá SSH, Sakamoto H, Pinto PPN, Battistella LR. Validation of the Brazilian version of the Functional Independence Measure. Acta Fisiátrica. 2004; 11(2):72-6. Doi: 10.5935/01047795.20040003

13) Talmelli LFS, Vale FAC, Gratão ACM, Kusumota L, Rodrigues RAPR. Alzheimer's disease: functional decline and stage of dementia. Acta Paul Enferm. 2013; 26(3):219-25.

14) World Health Organization. Measuring Health and Disability: Manual for WHO Disability Assessment Schedule (WHODAS 2.0). Geneve: WHO; 2010.

15) Silveira C, Parpinelli MA, Pacagnella RC, Camargo RS, Costa ML, Zanardi DM, Ferreira EC, Santos JP, Hanson L, Cecatti JG, Andreucci CB. Cross-cultural adaptation of the World Health Organization's Disability Assessment Scale (WHODAS 2.0) into Portuguese. Revista da Associação Médica Brasileira. 2013; 59(3):234-240. Doi: 10.1016/j.ramb.2012.11.005, PMid: 23684209

16) Baars BJ, Franklin S, Ramsoy TZ. Global workspace dynamics: cortical "blinding and propagation" enables conscious contents. Frontiers Psychology. 2013; 4, (200). Doi: 10.3389/fpsyg.2013.00200

17) Sakurada T, Ito K, Gom H. Bimanual motor coordination controlled by cooperative interactions in intrinsic and extrinsic coordinates. The European Journal of Neuroscience. 2016; 43(1):120-30. PMid: 26540267, PMCid: PMC4738419

18) Laban R. Domínio do Movimento. São Paulo: Summus, 1978. 
19) Groff, Ed. Laban Movement Analysis: charting the ineffable domain of human movement. Journal of Physical Education, Recreation \& Dance. 1995; 66(2):27-30. Doi: 10.1080/07303084.1995.10607038

20) Guimarães MC. Rudolf Laban: uma vida dedicada ao movimento. In: MOMMENSOHN, M. \& PETRELLA, P (Orgs.). Reflexões sobre Laban, o mestre do movimento. São Paulo: Summus, 2006.

21) Teixeira-Machado L. Dance therapy in autism: a case report. Fisioterapia e Pesquisa. 2015; 22(2):205-11. Doi: 10.590/1809-2950/11137322022015

22) Koch S, Kunz T, Lykou S, Cruz R. Effects of dance movement therapy and dance on health-related psychological outcomes: A meta-analysis. The arts in psychotherapy. 2014; 41(1):46-64. Doi: 10.1016/j.aip.2013.10.004

23) Le Breton D. “O inapreensível do corpo”. Antropologia do corpo. $4^{\text {th }}$ ed. Petrópolis: Vozes. 2016:15-34.

24) Foucault M. A Ordem do Discurso. 14 Edição. Editora Loyola, 2005.

25) Durkheim É. Formes élémentaires de 1 avie religieuse. Paris: PUF, 1968; p.368ss.

26) Mauss M. "As técnicas do corpo". In: Mauss, Marcel. Sociologia e antropologia. São Paulo: Cosac Naify. 2003:399-422. 